Article

\title{
Multi-Channel-Based Microgrid for Reliable Operation and Load Sharing
}

\author{
Ali Elrayyah and Sertac Bayhan * \\ Qatar Environment and Energy Research Institute, Hamad Bin Khalifa University, Doha 34110, Qatar; \\ aelrayyah@hbku.edu.qa \\ * Correspondence: sbayhan@hbku.edu.qa; Tel.: +974-4454-7188
}

Received: 6 May 2019; Accepted: 28 May 2019; Published: 30 May 2019

\begin{abstract}
This paper presents a novel approach to distribute available power among critical and non-critical loads in microgrids. The approach is based on supplying power over a number of channels with distinguishable frequencies where loads could be served by these channels according to their level of importance. The multi-channel scheme not only offers flexibility to supply loads but also to share power among adjacent microgrids. The control system, which can deal with multi-channel scheme, is presented and different applications that can be offered whereby are discussed. The number of channels that can be supplied by any inverter is determined based on the parameters of the used filter. Moreover, the power exchange efficiencies over the active channels at various power levels are determined and approximated formulas for quick evaluation are presented. To verify the proposed solution performance, simulation and experimental studies were performed. The obtained results demonstrate the effectiveness of using multi-channel scheme for power exchange in microgrid and also confirm the accuracy of the provided formula related to power exchange efficiencies.
\end{abstract}

Keywords: multi-channel power exchange; microgrid; power electronics-based source/loads; distributed generators; critical loads; uninterruptable power supply; power exchange efficiency

\section{Introduction}

The development of the microgrid concept has become an important element in the of the future utility grids and a priority in many countries due to its considerable environmental, economic, and social benefits. An increase of microgrid deployment rate will play an important role to meet future electricity demands without significant investment in new power plants. The main advantage of the microgrid is that it operates either in islanded mode or grid-connected mode. On the other hand, the power generation capacity of the distributed generators (DGs) is limited and it is often not sufficient to meet load demand in the islanded mode of operation. Therefore, a control system should be designed to ensure the continuity of the energy at critical loads (hospitals, data centers, etc.) and special attention should be paid for such loads in the islanded operation [1].

There has been extensive research and development in technologies, methods, and systems to secure a reliable power supply for various electric loads (critical and non-critical loads) [2]. In some aspects of their operation, microgrid systems still rely on the same operating principles that were established over a century ago [3]. Considering the advancement in power electronics technologies, loads and sources which are based on power electronics systems are expected to have significant share within power systems in near future [4-6]. Power electronics-based sources and loads can thus provide new means to revolutionize power exchange in microgrids.

One principle that remains rooted in microgrids is the non-differentiable sharing of power among loads. In all power systems, sources set up a single channel voltage and loads draw power from that channel. This indicates that critical as well as non-critical loads extract their need using the same 
approach. It would be very useful if there was an ability to enable sources to limit the supplied power to serve critical loads when there is a lack of sufficient power supply. However, sources cannot discriminate among the loads in these cases. There are solutions to address this problem which are based on establishing centralized energy management systems (EMSs) that monitor the whole system and adjust the loads accordingly $[7,8]$. However, EMSs could be challenging to establish especially when addressing large systems [9]. Moreover, the dynamics in the future inverter-based system could be too fast to be accommodated by such centralized-based systems [10]. The power packet presented in [11] proposes a scheme for power switching from one source to a specific load similar to the concept of circuit switching used in old telephone networks. This approach could be very effective but it requires a number of parallel lines and it needs high synchronization between switches.

In this paper, multi-channel-based microgrid is proposed to enhance system reliability and power sharing flexibility. The concept of using more than one frequency channel in microgrid is used in [12]. However, that concept is limited to superimposing a small AC signal driven by droop relation on a DC microgrid to ensure proper sharing among sources. The idea is further extended in [13] to manage power sharing among interlinking converters that transfer power between AC and DC sides of hybrid power systems. The approach proposed in this paper aims to use multi-channel not as communication mean, but rather to achieve an intelligent power exchange within/among microgrids.

The paper is organized as follows. The structure, operation, and control of multi-channel-based microgrid are discussed in Section 2 while the control of the sources and loads within that system are covered in Section 3. Section 4 provides theoretical analysis for the number of channels that can be used within a microgrid and estimate for the associated losses. The effectiveness of the proposed concept is demonstrated through simulation and experimental studies covered in Section 5. Finally, the paper is concluded in Section 6.

\section{The Proposed Multi-Channel-Based Microgrid Structure and Operation}

Figure 1 illustrates a conceptual diagram of the proposed multi-channel-based microgrid. Power sources-renewable-based or energy storage — can generate power at different frequency channels (50, 100, and $200 \mathrm{~Hz}$ in this case) through power electronics converters. The loads, either non-critical or critical, can then tune their power reception to one of more of these channels. The power sources can limit their supply to channels devoted to critical loads such that their power supply service is maintained uninterrupted when overloading occurs. The ability to exchange power over number of channels is not limited to individual sources and loads, but rather it can take place between subsystems such as microgrids.

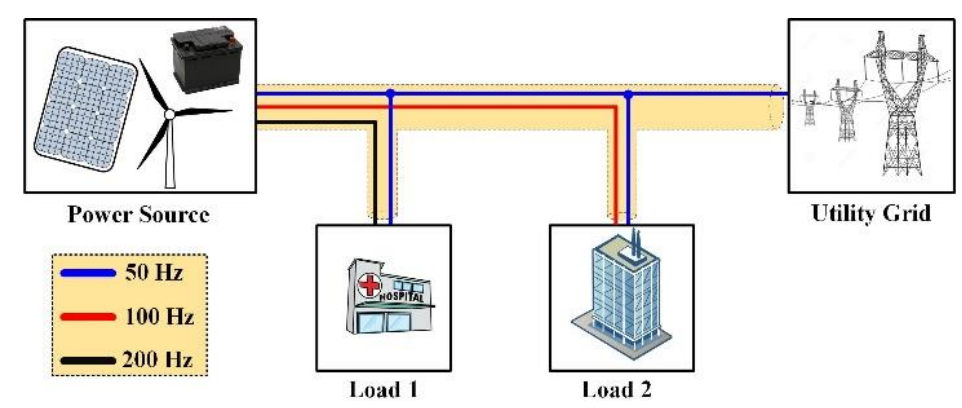

Figure 1. The conceptual diagram of the proposed multi-channel-based power system.

Fortunately, power electronics converters can support multi-channel-based power exchange with merely changes in their control algorithms and no hardware modification. Although the generated voltage signals contain harmonics, these are injected deliberately for power distribution in this application. To block the harmonic components, serial compensators (SCs), which are inverters connected in series with line to apply compensating voltages, are employed. The SCs can also be used in exchanging power among adjacent power systems. The operation of SCs is similar to the electric 
springs proposed in [14]. Electric springs regulate voltage and perform demand response by adding voltage components in series with load branches [15]. However, the operational scope of SCs is wider than that of electric springs as it deals with several channels.

The single-line diagram of the multi-channel microgrids is depicted in Figure 2. The system contains a number of multi-channel sources/loads as well as conventional ones. The power system is divided into subsystems. Grid and conventional loads are operated by a single frequency $(50 \mathrm{~Hz})$ while subsystems A and B can exchange power in multi-channel mode. In Figure 2, subsystem A can supply power in three channels; grid, local critical loads, and power exchange with subsystem B. It is well known that sharing power among interconnected subsystems in coordinated manner can provide several benefits for efficient and reliable system operation [16]. As shown in Figure 2, subsystem A can secure sufficient supply to its critical loads and also designate some power to subsystem $B$ that does not get interfered by other loads within the system. The connections between the subsystems are done through SCs which are operated to block the current flow of certain channels. A communication system can be used to set the various frequencies and to manage the power flow, but it is not critical for the system reliability.

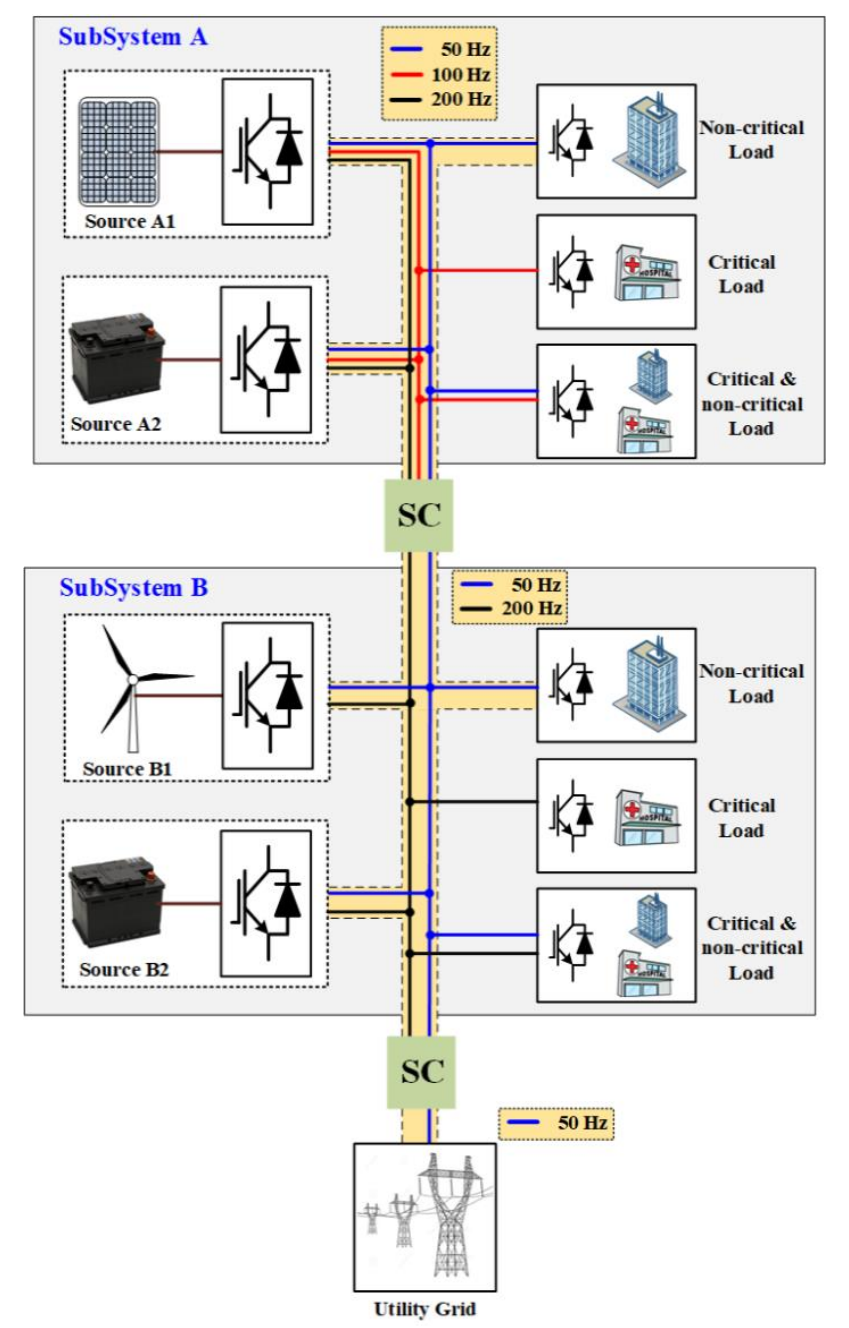

Figure 2. Single-line diagram of the multi-channel-based microgrids.

The power sources and loads in this system are connected to the network through power electronic converters. The line voltage in this power system composes of a number of frequency channels (harmonics). Each source can contribute power in any of these channels and likewise loads can receive power from any of these channels. Each source acts on parts of these channels as a voltage source 
where droop control can be applied. For the remaining channels, the source acts as a current source to feed some or zero current on each one of them.

Multi-channel-based microgrids shown in Figures 1 and 2 require changes in power system structure and the associated cost with these changes needs to be considered to evaluate the effectiveness of such approach. Fortunately, power electronics-based loads and sources are expected to have a larger share within microgrids in the future. As the following section shows, only changes in the inverter control logic are required to apply a multi-channel power exchange which does not impose any extra hardware cost. The need for SCs, on the other hand, might require an investment in additional hardware. However, the deployment of series compensating devices is expected to increase within distribution systems to accommodate for renewable energy sources integration [17]. Accordingly, the hardware components needed to apply the multi-power exchange are expected to be available within microgrids in the near future which opens the doors for this scheme to be utilized once the appropriate control logic is developed.

\section{Control of Multi-Channel Sources and Loads}

The control system of a multi-channel source is shown in Figure 3. Central controllers manage the various sources by informing them about the active channels and the amount of power that needs to be supplied in each channel. The phase locked loop block (PLL) in each source monitors the magnitude and angle of each channel to:

- Synchronize output voltage with active channels;

- Perform voltage/current control tasks and;

- Detect an activation/deactivation action in the system.

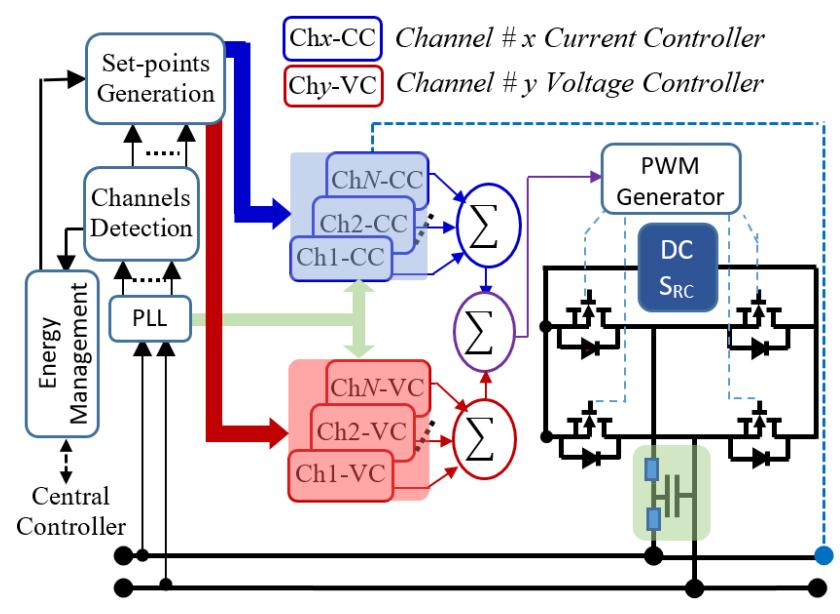

Figure 3. The block diagram of the control system of a source in a multi-channel-based power system.

Based on the applied operation scheme, the channels angles detected by the PLL are sent to current and/or voltage controllers to supply power through those channels. The outputs of these controllers are then combined to drive the inverter switches through the power width modulation (PWM) generator.

The multi-channel loads on the other hand operate as current sinks. In general, some channels could be defined for critical services and the other are for non-critical ones. Any load can then consume the power it needs for its essential functions from the critical channels, while it consumes the remaining demand from the other ones. The SC control structure is shown in Figure 4. This system works merely as a current source inverter where it adjusts the current at all channels that need to be blocked to zero. However, it does not contribute any voltage at the channel allowed to pass. 


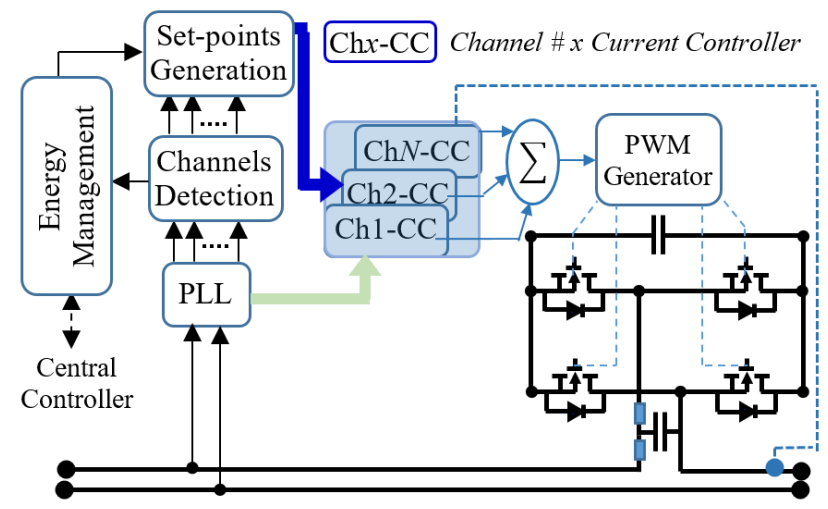

Figure 4. The block diagram of serial compensator control system.

The flowchart of multi-channel operation is given in Figure 5 . The cycle starts by updating the list of active channels based on commands sent from central controller. The controller then identifies active channels in the line through the PLL block. The importance of this step is of two folds. First, it is needed to identify the frequency and phase of each channel. Second, the PLL can detect channels' activation/deactivation immediately as it does not have to wait for command from the central controller. The control system then updates the power flow in various channels. Generally, every source needs to maintain a certain power reserve to accommodate for demand increase. As the sources supply more power, this reserve might not be maintained. To recover the required reserve level, sources can perform the following steps:

- If the source acts as a current source in some channels, it can reduce the supply to them till the reserve is maintained;

- If zero power is reached in current fed channels, sources can turn low-priority voltage-supplied channels into the current fed type and limit the current to a value that maintains the required reserve;

- If only high priority channels are active, sources can send a notification to central management unit to drop some of these channels;

- Voltage control loops and current control loops will then be updated based on their respective set-points and their combined output will be sent to the PWM controller that drives the switches.

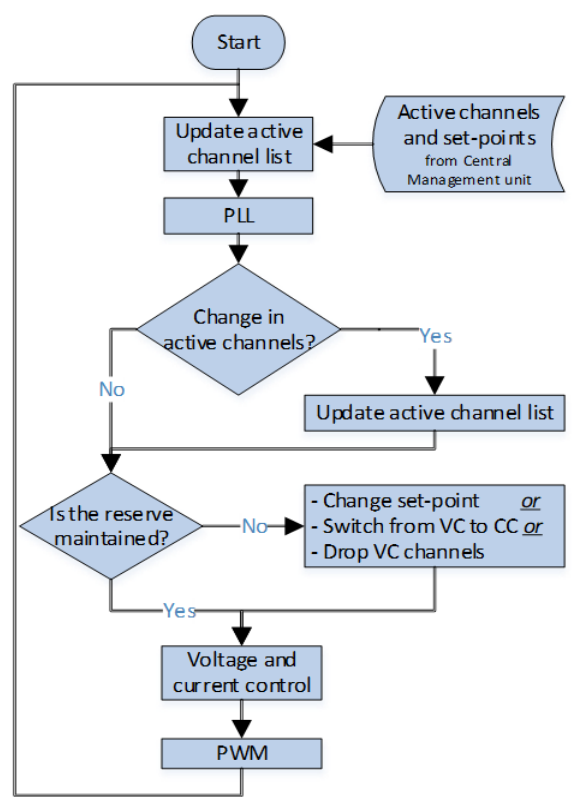

Figure 5. The flowchart of multi-channel operation scheme. 
The SC operates similar to the sources without the part related to maintaining the reserve. After detecting the active channels, the currents at blocked channels are set to zero through their current controller operation.

\section{Determination of Number and Frequencies of Usable Channels}

Activated frequencies in multi-channel systems cannot be arbitrarily selected. For effective operation, there must be clear separations between their frequencies, otherwise the PLL may not be able to identify them accurately. Moreover, even if the channel frequencies are selected with reasonable difference among them, their higher-order harmonics might not be distinguishable from one another. In this paper frequencies that meet these requirements are selected as:

$$
f_{k}=2^{k-1} f_{L}, k=1,2, \ldots, k_{m x}
$$

where $f_{L}$ is the frequency of the fundamental channel, $f_{k}$ is the frequency of the $k^{\text {th }}$ channel, and $k_{m x}$ is the number of channels that can be activated in the power zone. Since merely odd harmonics are experienced in power systems [18], there is at least $50 \mathrm{~Hz}$ separation between the harmonics components of the channels indicated in Equation (1).

Different types of filters could be used for multi-channel inverters. However, LCL is considered in this paper since in requires relatively low inductors. Having a low inductor is very important to reduce its associated voltage drop. As indicated in [19], the resonance frequency of LCL filter needs to satisfy

$$
10 f_{L}<f_{\text {res }}<\frac{1}{2} f_{\text {sw }}, \text { fres }=\frac{1}{2 \pi} \sqrt{\frac{L_{1}+L_{2}}{L_{1} L_{2} C}}
$$

where $f_{\text {res }}$ is the resonance frequency of the LCL filter and $f_{s w}$ is the switching frequency. In the case of a multi-channel system, Equation (2) implies

$$
10 \times 2^{k_{m x}-1} f_{L}<f_{\text {res }}<\frac{1}{2} f_{\text {sw }}
$$

Equation (3) leads to the following relation

$$
2^{k_{m x}-1}<\frac{f_{s w}}{20 f_{L}} \rightarrow k_{m x}<3.32\left(\log \left(\frac{f_{s w}}{f_{L}}\right)-1\right)
$$

Accordingly, for a $50 \mathrm{~Hz}$ system with a $20 \mathrm{kHz}$ switching frequency, $k_{m x} \leq 5$.

To investigate the other constraints for acceptable value of $k_{m x}$, the following information needs to be provided for each channel: frequency $\left(f_{k}\right)$, rated power $\left(P_{m x, k}\right)$, and voltage magnitude $\left(V_{m x, k}\right)$. Consider a multi-channel inverter system shown in Figure 6 where power is transferred over the $k^{\text {th }}$ channels from a source to a load. As multi-channel systems are based on power electronics sources and loads, it is more effective to transfer only real power as the reactive power needed by loads could be provided by their local inverters. Accordingly, the line voltage $V_{o, k}$ and output current $i_{o, k}$ are assumed to be in phase. The voltage at the capacitor $V_{C, k}$ of the source inverter is given by

$$
V_{C, k}=V_{o, k}+j \omega_{k} L_{2} i_{o, k} \rightarrow\left|V_{C, k}\right|=\sqrt{V_{o, k}^{2}+\left(\omega_{k} L_{2} i_{o, k}\right)^{2}} \approx V_{o, k}
$$

The approximation in Equation (5) is done to simplify the analysis and since the voltage drop $\omega_{k} L_{2} i_{o, k}$ should to be very small in comparison with $V_{o, k}$ for reasonable power transfer. The reactive power supplied to the capacitor $\left(Q_{C, k}\right)$ can then be approximated by

$$
Q_{C, k} \approx-\omega_{k} C V_{o, k}^{2}
$$


The current that flows over the inductor $L_{1}\left(i_{i, k}\right)$ accordingly becomes:

$$
i_{i, k} \approx i_{o, k}+j \omega_{k} C V_{o, k}
$$

By summing the reactive power supplied to $L_{1}, L_{2}$, and $C$ total reactive power $Q_{k}$ supplied by the inverter can then be written as:

$$
\begin{aligned}
& Q_{k} \approx-\omega_{k} C V_{o, k}^{2}+\omega_{k}\left(\omega_{k} C V_{o, k}\right)^{2} L_{1}+\omega_{k} i_{o, k}^{2}\left(L_{1}+L_{2}\right) \\
& =\omega_{k}\left(-C V_{o, k}^{2}\left(1-\omega_{k}^{2} L_{1} C\right)+P_{o, k}^{2} / V_{o, k}^{2}\left(L_{1}+L_{2}\right)\right)
\end{aligned}
$$

Assuming $R_{L 1}, R_{L 2}$, and $R_{C}$ are the resistances of $L_{1}, L_{2}$, and $C$ the power transmission efficiency $\left(\eta_{k}\right)$ from source to load could be estimated using the relation:

$$
\eta_{k}=\frac{P_{o, k}}{P_{o, k}+2\left(\frac{P_{o, k}}{V_{o, k}}\right)^{2}\left(R_{L 1}+R_{L 2}\right)+2\left(Q_{C, k} / V_{o, k}\right)^{2}\left(R_{L 1}+R_{C}\right)}
$$

Equations (8) and (9) can be used to determine whether certain power could be transferred over a specific power depending on the associated reactive power and losses.

Another constraint for multi-channel inverter is related to its DC bus voltage $\left(V_{D C}\right)$. The DC bus voltage needs to be high enough to generate the required output voltage at all channels. The appropriate value for the DC bus voltage depends on the phase angle of the various channels. When the phase angles of the channels are allowed to be arbitrary set, the DC bus voltage needs to satisfy the following relation

$$
\sqrt{2} \sum_{k=1}^{k_{m x}} V_{m x, k}<V_{D C}
$$

Allowing the channels to have any phase is very important for certain applications. For example, when droop control is implemented over the various channels, the exact channel frequency varies slightly around the nominal channel frequency and so does the phase angle. In this case Equation (10) needs to be satisfied. However, in another application the master source could be responsible to set the line voltage $\left(V_{L}\right)$. In this case, the channel frequencies and phases can be maintained at fixed values set by the master source as:

$$
V_{L}=\sqrt{2} \sum_{k=1}^{k_{m x}} V_{m x, k} \sin \left(2^{k-1} \pi f_{L} t+\phi_{k}\right)
$$

If $\phi_{k}$ of the various channels do not have the same value, the positive and negative half cycles will have different shapes. It is therefore preferred that all channels have the same value of $\phi_{k}$ which can be taken as zero. To determine the peak voltage of $V_{L}$ in this case, let $\pi f_{L} t$ be defined as $\theta$, then the following equation needs to be solved for $\theta$ in the range $[0, \pi]$

$$
\frac{d V_{L}}{d \theta}=\sqrt{2} \sum_{k=1}^{k_{m x}} 2^{k-1} V_{m x, k} \cos 2^{k-1} \theta=0
$$

The values of $\theta$ that solves Equation (12) can then be used to determine the peak voltage. For example, consider the case of three channels with RMS values of $V_{m x, 1}, V_{m x, 2}$ and $V_{m x, 3}$ given by $V_{m}$, $0.5 V_{m}$, and $0.5 V_{m}$, respectively. Solving Equation (12) yields a peak voltage for $V_{L}$ as $1.37 \sqrt{2} V_{m}$. The DC bus voltage in this case can be taken as $1.37 \sqrt{2} V_{m}$ representing $68 \%$ of the value set by Equation (10). This indicates that, depending on the intended application, selecting the right setting for the channel frequencies and phases can have significant impact in components' sizing and design.

Besides the constraints related to switching/resonant frequencies, reactive power supply, and DC bus voltage, ripple in inverter current and voltage drop over the inductors are usually considered 
while designing LCL filters. The ripple in the inverter output current $\Delta i_{L 1}$ in unipolar PWM switching is given by [20]:

$$
\Delta i_{L 1}=\frac{V_{D C} L_{1}}{8 f_{s w}}
$$

$L_{1}$ must then be selected to maintain $\Delta i_{L 1}$ below the required limit. This value of $L_{1}$ can be used to estimate the voltage drop of the LCL filter. The voltage drop over the filter inductors is usually needed to be kept below a certain value for proper voltage regulation. For the $k^{\text {th }}$ channel, the voltage drop over the filter inductors can be written as:

$$
\Delta V_{o, k}=V_{o, k}-m_{a, k} V_{D C} \approx j \omega_{k} L_{1}\left(i_{C, k}+i_{o, k}\right)+j \omega_{k} L_{2} i_{o, k}=\omega_{k}^{2} L_{1} C V_{o, k}+j \omega_{k}\left(L_{2}+L_{1}\right) i_{o, k}
$$

$m_{a, k}$ is the inverter modulation index. The term $V_{o, k} \omega_{k}^{2} L_{1} C$ has a 180 degree phase shift from $V_{o, k}$ and it is added to the inverter output voltage while the term $j \omega_{k} i_{o, k}\left(L_{1}+L_{2}\right)$ has a 90 degree phase shift from $V_{o, k}$. Based on any requirement for the line voltage drop, the relation in Equation (14) can be used to check the possibility of activating a certain channel with the power system under consideration.

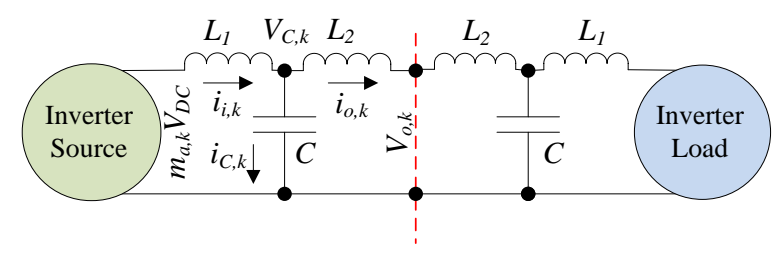

Figure 6. LCL filter for multi-channel inverter.

\section{Simulation and Experimental Studies}

To verify the effectiveness of the proposed multi-channel-based power systems, simulation and experimental studies have been conducted. In the experimental setup, a Chroma regenerative grid simulator is used to set up the three channels. Since the grid simulator can absorb power it is used to set the line voltage and to emulate loads in the system. Two sources are then used to supply power over the various channels. Each of the sources is supplied by Magna-Power power supply and it uses IAP inverter and controlled by TMS320F28335 DSP. The system is also simulated using Matlab to analyze its efficiency at different power levels. The considered diagram for the microgrid is shown in Figure 6 where the source and load inverters have identical parameters which are listed in Table 1 . The resistors $R_{L 1}, R_{L 2}$, and $R_{C}$ are assumed to be connected in series with $L_{1}, L_{2}$, and $C$, respectively.

Table 1. Inverter parameter of the considered system.

\begin{tabular}{cccc}
\hline Parameter & Value & Parameter & Value \\
\hline$C$ & $22.5 \mu F$ & $R_{C}$ & $1.0 \Omega$ \\
\hline$L_{1}$ & $0.5 \mathrm{mH}$ & $R_{L 1}$ & $0.5 \Omega$ \\
\hline$L_{2}$ & $0.5 \mathrm{mH}$ & $R_{L 2}$ & $0.5 \Omega$ \\
\hline Voltage & $120 \mathrm{~V}$ & $f_{L}$ & $50 \mathrm{~Hz}$ \\
\hline
\end{tabular}

From Table 1, $f_{\text {res }}=2.1 \mathrm{kHz}$ indicating that $k_{m x}$ can at most be 3 . For source inverter, Figure $7 \mathrm{a}$ compares the simulated per unit reactive power and the one provide by Equation (8). For the small value of $P_{o, k}$ the term $-\omega_{k} C V_{o, k}^{2}$ is dominant, especially for higher-order channels, and the inverter supplies more reactive power. However, as $P_{o, k}$ increases, the value of $\omega_{k} P_{o, k}^{2} / V_{o, k}^{2}\left(L_{1}+L_{2}\right)$ causes the supplied reactive power to decrease. Note that the power ranges that correspond to high amounts of reactive power need to be avoided to minimize operating losses. This can be observed clearly in Figure $7 \mathrm{~b}$, since when channel 3 supplies less than $0.5 \mathrm{~kW}$, the transmission efficiency is relatively 
low. When the transmitted amount of power increases, the losses due to the term $\left(Q_{k} / V_{o, k}\right)^{2}\left(R_{L 1}\right)$ decrease, while that of $\left(P_{o, k} / V_{o, k}\right)^{2}\left(R_{L 1}+R_{L 2}\right)$ becomes dominant and hence the efficiencies of all channels become comparable and they all decrease by the same rate.

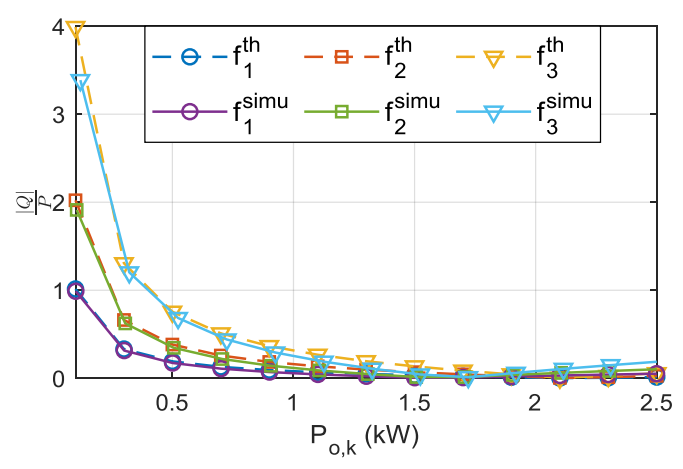

(a)

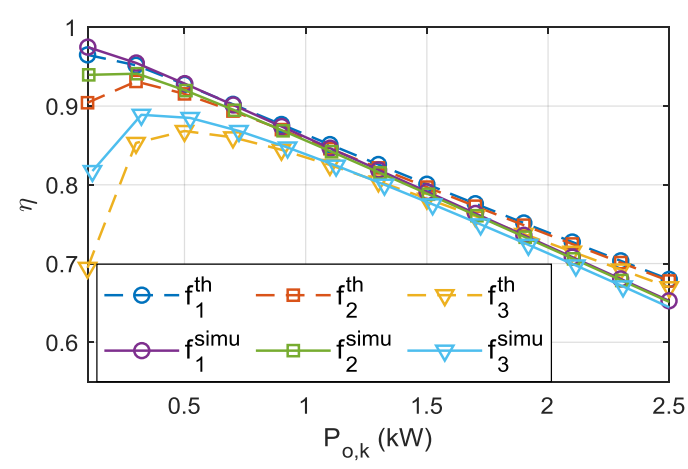

(b)

Figure 7. Reactive power supplied by multi-channel inverter to LCL components over the various channels: (a) Reactive power supply using Equation (8) and simulation study; (b) power exchange efficiency over various channels.

The microgrid system in Figure 8 is considered for experimental studies. Two sources (Src1 and Src2) feed power as current sources to loads in three channels ch1, ch2, and ch3 which have the frequencies 50, 100, and $200 \mathrm{~Hz}$, respectively. The line voltage in the system is given by:

$$
v_{L}=V_{1} \sin \omega_{1} t+V_{2} \sin \omega_{2} t+V_{3} \sin \omega_{3} t
$$

where $V_{1}=V_{2}=V_{3}=83$. Though all channels are generated by Chroma power simulator, ch1 is taken to represent the grid. Two case studies are considered where the sources feed power over the channels differently and the event of grid disconnection are analyzed.

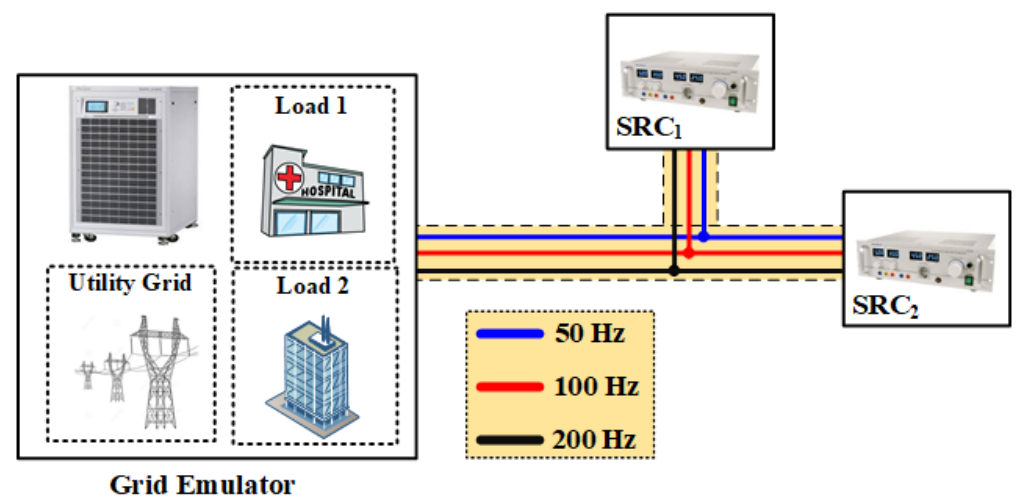

Figure 8. Considered microgrid for experimental study.

\subsection{Case Study I}

In the first case study, Src1 supplied $7 \mathrm{~A}$ at ch1 only while Src2 supplied $7 \mathrm{~A}$ in each of the three channels. This can be used in applications where the supply of Src1 is unreliable and thus is used to supply uncritical loads over ch1, while Src2 supplies critical loads that are served by ch2 and ch3 as well as uncritical ones in ch1. Figure 9a shows the line voltage and current supplied by the two sources. 


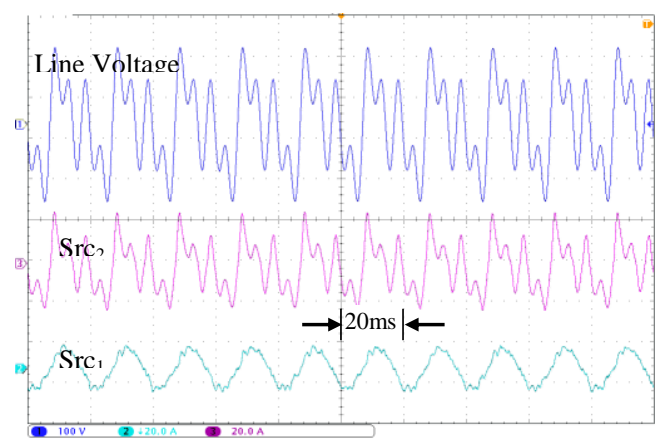

(a)

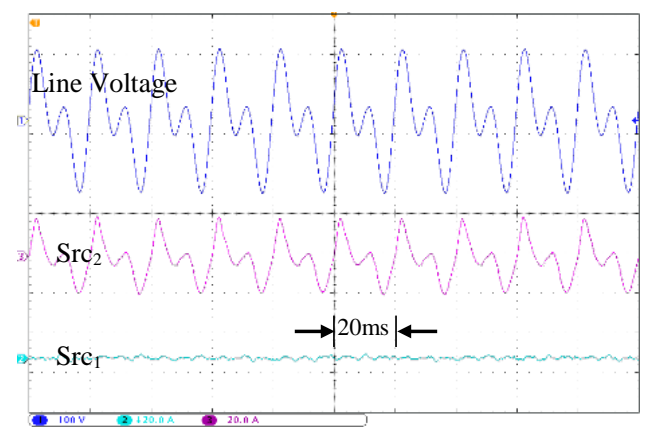

(c)

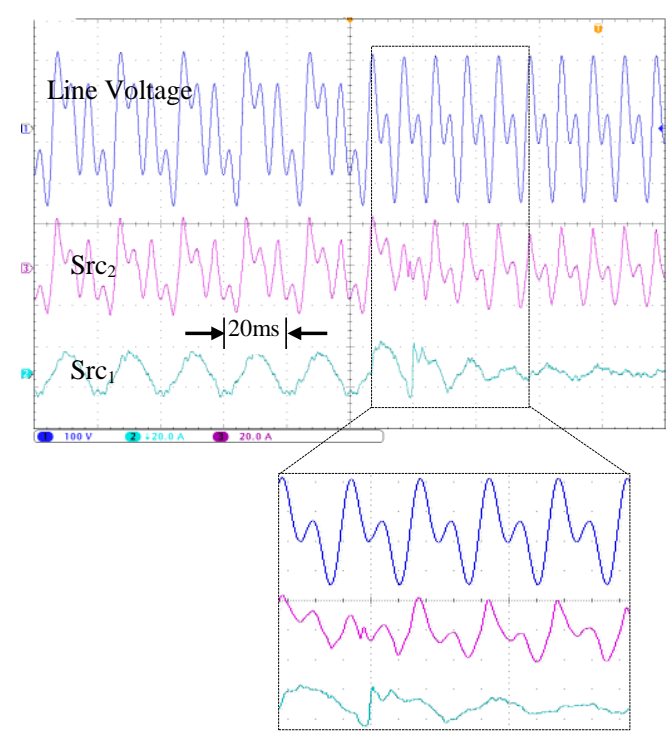

(b)

Figure 9. Experimental study sources' behavior in multi-channel power system after grid disconnection where one source supplies power to the grid channel only while the other feeds power to all channels; (a) Src1 feeds power to ch1 and Src2 feeds power over all channels; (b) grid disconnection causing elimination of ch1; (c) Src1 and Src2 output currents after grid disconnection.

At $\mathrm{t}=4.5 \mathrm{~s}$, the grid disconnection was imposed to analyze the system performance. The controllers of Src1 and Src2 could detect this event locally and eliminate any power supply over ch1. The line voltage and sources currents waveforms reflected that as shown in Figure 9b. Eventually, the line voltage and sources current were adjusted to feed the loads that were served by ch2 and ch3 as shown in Figure 9c.

The power and energy fed by the two sources for case study I are demonstrated in Figure 10. Before $t=4.5 \mathrm{~s}$, Src2 fed three times the power of Src1 as it was active over three channels. However, after the grid disconnection, the power supplied over ch1 was dropped, which represented all the power supplied by Src1 and one third of Src2 supplied power. This behavior could be very useful to enable sources to play different roles in supplying load-demand with fast and reliable responses to system changes and without explicit inter-controllers communication.

\subsection{Case Study II}

Case study II represents an application where certain critical loads need not to experience any disruption in their power supply. In this case, sources were configured such that one source (Src2) was exclusively used to serve critical loads in ch2 and ch3 while Src1 participated a guaranteed amount of power to critical loads, with any extra power being fed to ch1. Originally, as Figure 11a shows, Src1 supplied 7 A over the three channels and Src2 supplied $7 \mathrm{~A}$ in each of ch2 and ch3. At $\mathrm{t}=4.5 \mathrm{~s}$, grid disconnection was imposed which eliminated ch1 from the line voltage. As shown in Figure 11b, Src1 could respond to this event by adjusting their current at ch1 to zero without any major disturbance in the sources current supply over ch2 and ch3. The system then maintained the operation under the new condition highlighted in Figure 11c. 

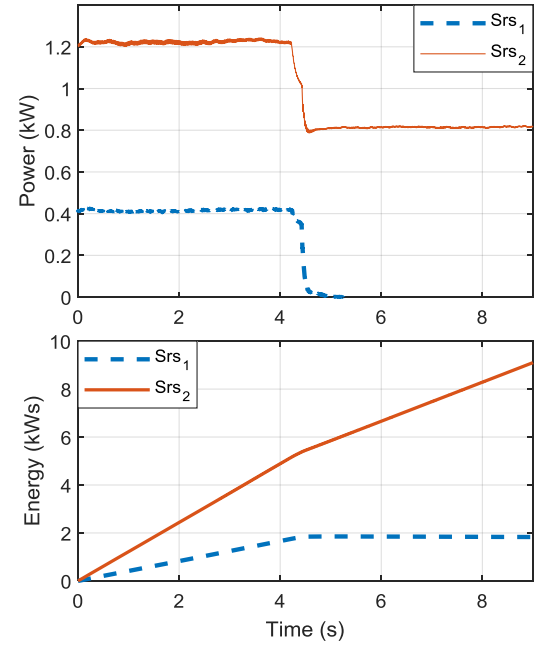

Figure 10. Power and energy supplied by Src1 and Src2 in case study I.

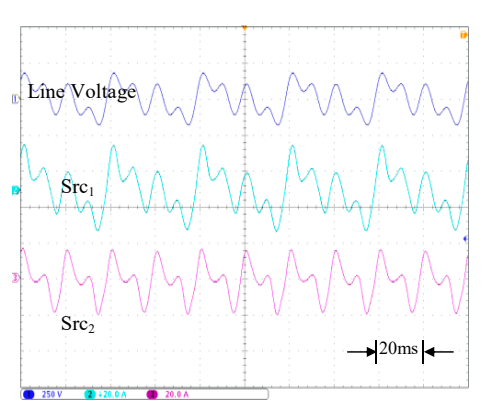

(a)

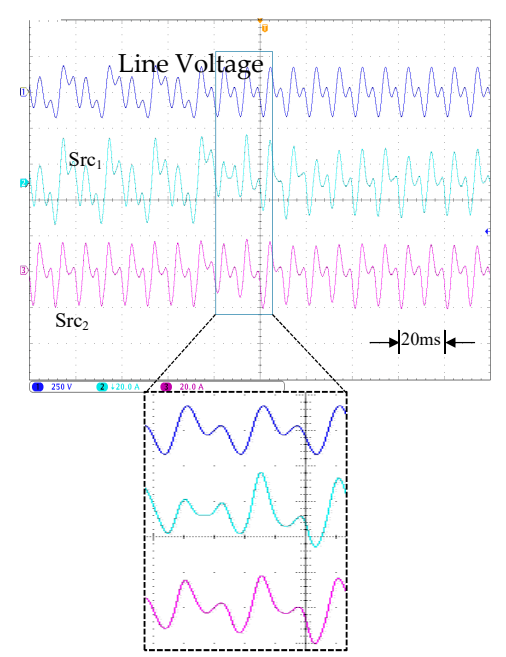

(b)

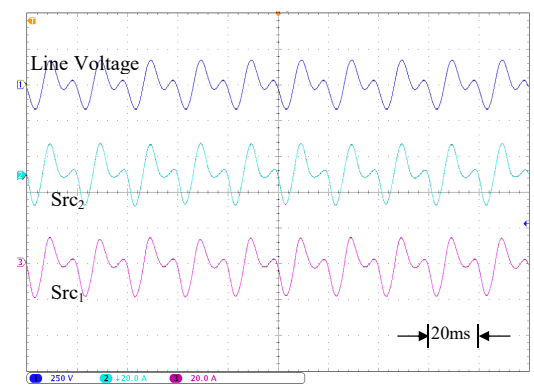

(c)

Figure 11. Experimental study sources behavior in multi-channel power system after grid disconnection where one sources supply power to all channels while the other supplies power merely to critical loads on non-grid supplied channels; (a) Src1 feeding power in ch2 and ch3 and Src2 feeding power over all three channels; (b) Grid disconnection casuing elimination of ch1, (c) Src1 and Src2 output currents after grid disconnection.

The power and energy fed by the two sources are demonstrated in Figure 12. The power supply of Src2 was maintained at the same value throughout the experiment duration as it goes in its entirety to critical loads. On the other hand, one third of the Src1 supplied power was withdrawn after $t=4.5$ since that power was used to feed uncritical loads or to be fed back to the grid. 

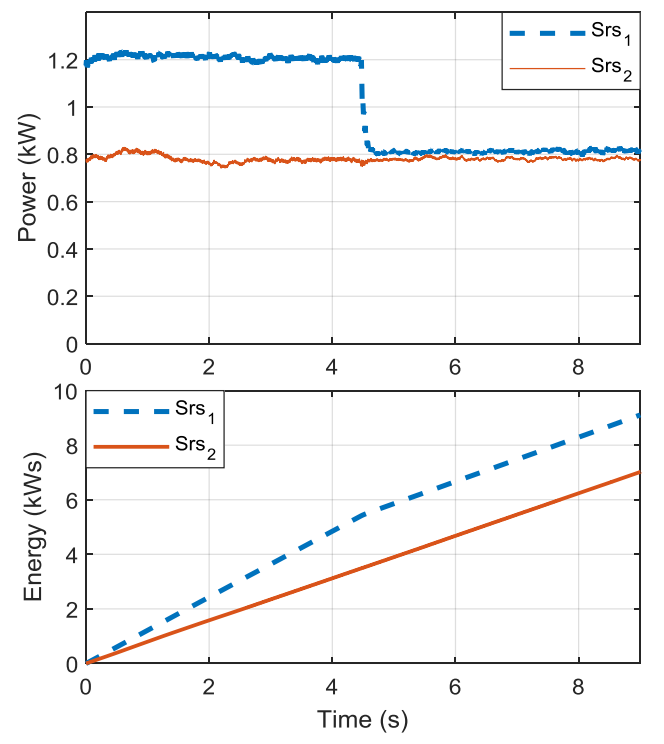

Figure 12. Power and energy supplied by Src1 and Src2 in case study II.

\section{Conclusions}

The use of sources that supply power over a number of frequencies in multi-channel microgrids provides many benefits to power systems. A multi-channel-based power supply helps maintain an uninterruptable power supply to critical loads, enhances system reliability, and allows more liberalized power trading among adjacent microgrids. The paper shows the viability of supplying power over more than one channel through the proper configuration of voltage and current controllers. The parameters of an inverter filter determine the maximum number of channels that can be supplied by that inverter. Moreover, supplying low power at higher-order channels has poor efficiency as a significant amount of reactive power is supplied in these cases to the reactive components of the filter. The formulas provided in the paper for the allowed number of channels and power exchange efficiencies assist in determining the effectiveness of applying the concept of a multi-channel scheme in any microgrid. The paper focuses on introducing the concept of a multi-channel power exchange, its implementation scheme, some of the related advantages, and discussion of its limitations. In future publications, we plan to cover and analyze in detail subjects such as system stability, controller design, and interactions among various channels.

Author Contributions: The research presented in this paper was a collaborative effort among both authors. A.E. and S.B. conceived, implemented, and got the results along with the paper write-up. A.E. and S.B. wrote the paper and discussed the results and revised the manuscript critically.

Funding: The publication of this article was funded by the Qatar National Library.

Acknowledgments: The publication of this article was funded by the Qatar National Library.

Conflicts of Interest: The authors declare no conflict of interest.

\section{References}

1. Li, J.; Liu, Y.; Wu, L. Optimal Operation for Community-Based Multi-Party Microgrid in Grid-Connected and Islanded Modes. IEEE Trans. Smart Grid 2018, 9, 756-765. [CrossRef]

2. Liu, M.; Li, W.; Wang, C.; Polis, M.P.; Wang, 1.; Li, J. Reliability Evaluation of Large Scale Battery Energy Storage Systems. IEEE Trans. Smart Grid 2017, 8, 2733-2743. [CrossRef]

3. Tan, D.; Novosel, D. Energy Challenge, Power Electronics \& Systems (PEAS) Technology and Grid Modernization. CPSS Trans. Power Electron. Appl. 2017, 2, 3-11.

4. Huber, J.E.; Kolar, J.W. Applicability of Solid-State Transformers in Today's and Future Distribution Grids. IEEE Trans. Smart Grid 2018, 10, 317-326. [CrossRef] 
5. Xu, S.; Huang, A.Q.; Burgos, R. Review of Solid-state Transformer Technologies and Their Application in Power Distribution Systems. IEEE Trans. Emerg. Sel. Top. Power Electron. 2013, 1, 186-198.

6. Che, L.; Shahidehpour, M. DC Microgrids: Economic Operation and Enhancement of Resilience by Hierarchical Control. IEEE Trans. Smart Grid 2014, 5, 2517-2526.

7. Farzin, H.; Fotuhi-Firuzabad, M.; Moeini-Aghtaie, M. Role of Outage Management Strategy in Reliability Performance of Multi-Microgrid Distribution Systems. IEEE Trans. Power Syst. 2018, 33, 2359-2369. [CrossRef]

8. Lopes, J.A.P.; Moreira, C.L.; Madureira, A.G. Defining Control Strategies for Microgrids Islanded Operation. IEEE Trans. Power Syst. 2006, 21, 916-924. [CrossRef]

9. Han, H.; Hou, X.; Yang, J.; Wu, J.; Su, M.; Guerrero, J.M. Review of Power Sharing Control Strategies for Islanding Operation of AC Microgrids. IEEE Trans. Smart Grid 2016, 7, 200-215. [CrossRef]

10. Olivares, D.E.; Mehrizi-Sani, A.; Etemadi, A.H.; Cañizares, C.A.; Iravani, R.; Kazerani, M.; Hajimiragha, A.H.; Gomis-Bellmunt, O.; Saeedifard, M.; Palma-Behnke, R.; et al. Trends in Microgrid Control. IEEE Trans. Smart Grid 2014, 5, 1905-1919. [CrossRef]

11. Takahashi, R.; Tashiro, K.; Hikihara, T. Router for Power Packet Distribution Network: Design and Experimental Verification. IEEE Trans. Smart Grid 2015, 6, 618-626. [CrossRef]

12. Peyghami, S.; Mokhtari, H.; Blaabjerg, F. Autonomous Operation of a Hybrid AC/DC Microgrid with Multiple Interlinking Converters. IEEE Trans. Smart Grid 2018, 9, 6480-6488. [CrossRef]

13. Peyghami, S.; Mokhtari, H.; Loh, P.C.; Davari, P.; Blaabjerg, F. Distributed Primary and Secondary Power Sharing in a Droop-Controlled LVDC Microgrid with Merged AC and DC Characteristics. IEEE Trans. Smart Grid 2018, 9, 2284-2294. [CrossRef]

14. Hui, S.Y.R.; Lee, C.K.; Wu, F.F. Electric springs-A New Smart Grid Technology. IEEE Trans. Smart Grid 2012, 3, 1552-1561. [CrossRef]

15. Chen, X.; Hou, Y.; Hui, S.Y.R. Distributed Control of Multiple Electric Springs for Voltage Control in Microgrid. IEEE Trans. Smart Grid 2017, 8, 1350-1359. [CrossRef]

16. Wang, H.; Huang, J. Incentivizing Energy Trading for Interconnected Microgrids. IEEE Trans. Smart Grid 2018, 9, 2647-2657. [CrossRef]

17. Wang, J.; Wang, Z.; Xu, L.; Wang, Z. A Summary of Applications of D-FACTS on Microgrid. In Proceedings of the 2012 Asia-Pacific Power and Energy Engineering Conference, Shanghai, China, 27-29 March 2012; pp. 1-6.

18. Bidram, A.; Davoudi, A. Hierarchical Structure of Microgrids Control System. IEEE Trans. Smart Grid 2012, 3, 1963-1976. [CrossRef]

19. Reznik, A.; Simoes, M.G.; Al-Durra, A.; Muyeen, S.M. LCL Filter Design and Performance Analysis for Grid-Interconnected Systems. IEEE Trans. Ind. Appl. 2014, 50, 1225-1232. [CrossRef]

20. Ruan, X.; Wang, X.; Pan, D.; Yang, D.; Li, W.; Bao, C. Control Techniques for LCL-type Grid-connected Inverters; Springer: Berlin, Germany, 2018; pp. 31-61. 